\title{
Grid Equipment Malfunction Prediction of Association Rules Merging With Gray BP
}

\author{
Zhang $\mathrm{Hu}^{1, a}$,Wang Hongbin ${ }^{1, a}$, Li Feng ${ }^{1, a}$ \\ ${ }^{1}$ Electric Power Research Institute of Guangdong Power Grid Co., Ltd., Guangzhou \\ 510000,Guangdong Province, China \\ a Email: zhanghu_gd@sina.com
}

\section{Key Words: Power Grid, Power Feedforward, Robust Prediction, Dead-beat Control}

\begin{abstract}
In PV microgrid control system, the delay and filter inductance value change will influence the response speed, stability and grid-connected current distortion rate of system. Therefore, this article proposes a power feedforward robust prediction dead-beat grid-connected control method. The system's response speed is sped up by introducing power feedforward. The robust prediction dead-beat control method is proposed to be used on grid-connected control to strengthen robustness of system and reduce distortion of grid-connected current caused by control delay and inductance value deviation. It also analyzes Z-domain dead-beat control model and discusses influences of delay and inductance value on system stability. This article gives the design method of robust prediction dead-beat control and performs stability analysis according to transfer function within Z-domain, determines selection scope of control parameters. The simulation and experiment results prove the effectiveness of the proposed control method.
\end{abstract}

\section{Introduction}

Microgrid is a rising cutting-edge technology on the basis of new energy distributed generation which support large power grid with each other and is an effective method to increase energy supply efficiency of distributed generation. The future small and medium size smart distribution grid will have more distributed mircrogrid with new energy generation. At present, the fragile of large power grid is more and more prominent. Combining the key loads which are adjacent in locations into mircrogrids and designing appropriate circuit topology structure and control method will not only reduce cost of enhancing overall stability and power quality, but also will reduce economic loss caused by power cut.

\section{Grid Inverter Dead-beat Control}

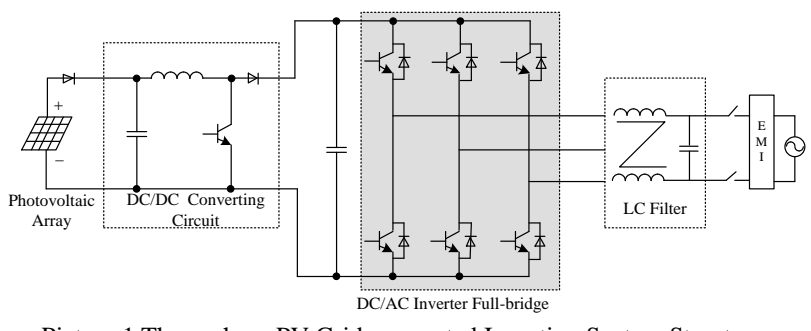

Picture 1 Three-phase PV Grid-connected Inverting System Structure

Picture 1 shows three-phase PV grid-connected inverting system structure frame which includes the DC/DC convertor in preceding stage and three-phase DC/AC grid-connected inverter. Given the voltage of three-phase grid is balanced and switch loss and breakover voltage drop of power switch parts is neglected, the PV grid-connected VSI system mathematical model established under two-phase static $\alpha \beta$ coordinate system according to Kirchhoff's law is

$$
\boldsymbol{u}_{g \alpha \beta}=\boldsymbol{v}_{g \alpha \beta}+L_{g} \frac{d \boldsymbol{i}_{g \alpha \beta}}{d t}+R_{g} \boldsymbol{i}_{g \alpha \beta}
$$

In the formula:ug $\alpha \beta$ is $\alpha \beta$ coordinate system component of grid voltage; $\operatorname{vg} \alpha \beta$ is $\alpha \beta$ coordinate system component of voltage at grid-connected VSI end; ig $\alpha \beta$ is $\alpha \beta$ coordinate system component of grid current; Lg is filter reactor of line side, Rg is equivalent resistance. 
According to instantaneous reactive power, the instantaneous active power, reactive power of three-phase symmetric system can be described as

$$
\left\{\begin{array}{l}
P_{g}=-1.5\left(u_{g \alpha} i_{g \alpha}+u_{g \beta} i_{g \beta}\right) \\
Q_{g}=-1.5\left(u_{g \beta} i_{g \alpha}-u_{g \alpha} i_{g \beta}\right)
\end{array}\right.
$$

In order to gain the change rate of instantaneous active power and reactive power, the differential treatment for formula (2) is as follows:

In formula: the ideal grid voltage change rate is

$$
\left\{\begin{array}{l}
\frac{d P}{d t}=-1.5\left(e_{\alpha} \frac{d i_{\alpha}}{d t}+i_{\alpha} \frac{d e_{\alpha}}{d t}+e_{\beta} \frac{d i_{\beta}}{d t}+i_{\beta} \frac{d e_{\beta}}{d t}\right) \\
\frac{d Q}{d t}=-1.5\left(e_{\beta} \frac{d i_{\alpha}}{d t}+i_{\alpha} \frac{d e_{\beta}}{d t}-e_{\alpha} \frac{d i_{\beta}}{d t}-i_{\beta} \frac{d e_{\alpha}}{d t}\right)
\end{array}\right.
$$

$$
\left\{\begin{array}{l}
\frac{d e_{\alpha}}{d t}=\omega|\boldsymbol{e}| \sin (\omega t)=-\omega e_{\beta} \\
\frac{d e_{\beta}}{d t}=\omega|\boldsymbol{e}| \cos (\omega t)=\omega e_{\alpha}
\end{array}\right.
$$

Until now, substituting formulas (1) and (4) into (3) can get instantaneous power change rate of grid-connected system, which is

$$
\left\{\begin{array}{l}
\frac{d P}{d t}=-\frac{3}{2 L}\left[|\boldsymbol{e}|^{2}-\left(e_{\alpha} v_{\alpha}+e_{\beta} v_{\beta}\right)\right]-\frac{R}{L} P-\omega Q \\
\frac{d Q}{d t}=-\frac{3}{2 L}\left[-\left(e_{\beta} v_{\alpha}-e_{\alpha} v_{\beta}\right)\right]-\frac{R}{L} Q+\omega P
\end{array}\right.
$$

Influences of Dead-beat Control on Stability

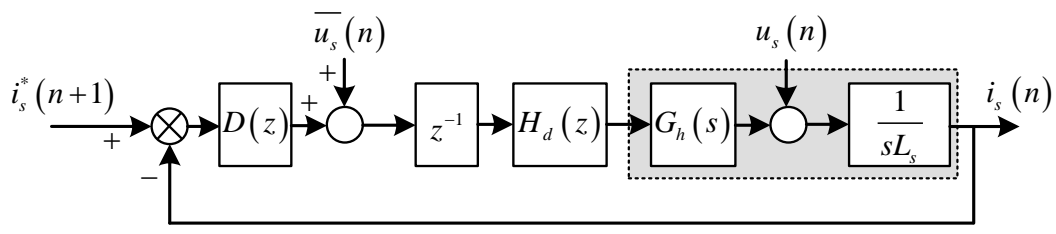

Picture 2 Two Electrical Power VSI Voltage Space Vector Distribution Diagram

Considering the control delay problem existing in actual digital processing system, Picture 2 gives calculation and Z-domain dead-beat control equivalent model of VSI system delay. Among it, zero-order holder $(\mathrm{ZOH})$ transfer function is

$$
G_{h}(s)=\left(1-e^{-T_{s} s}\right) / s
$$

Then Z-domain transfer function of zero-order holder and inductance is

$$
G_{d}(z)=\left(1-z^{-1}\right) Z\left[\frac{1}{s} \cdot \frac{1}{s L_{s}}\right]=\frac{T_{s}}{L_{s}} \cdot \frac{1}{Z-1}
$$

Because the transfer function of z-1 with delay link can be represented as e-Tds, the approximate value can be get by expanding Taylor series as follows:

$$
e^{-T_{d} s}=\frac{1}{1+T_{d} s+T_{d}^{2} s^{2} / 2+\cdots} \approx \frac{1}{1+T_{d} s}
$$

Given Ls is predicted value of grid-connected inductance value which has certain deviation with actual inductance value $\mathrm{Z}$. The deviation degree is balanced by inductance coefficient, that is, $\mathrm{kL}$ $=\mathrm{Ls} / \mathrm{L}$, then $\mathrm{D}(\mathrm{z})=\mathrm{kLLs} / \mathrm{Ts}$. Given the proportionality coefficient of PWM delay is $\mathrm{kT}=\mathrm{Td} / \mathrm{Ts}$, then the transfer function of Td with delay link is

$$
H_{d}(z)=\left[\left(1-k_{T}\right) z+k_{T}\right] / z
$$

In conclusion, it can be got that the system closed-loop transfer function of td with delay link is 


$$
G(z)=\frac{D(z) z^{-1} H_{d}(z) G_{d}(z)}{1+D(z) z^{-1} H_{d}(z) G_{d}(z)}
$$

At this time, the closed-loop system feature function is

$$
z^{3}-z^{2}+k_{L}\left(1-k_{T}\right) z+k_{L} k_{T}=0
$$

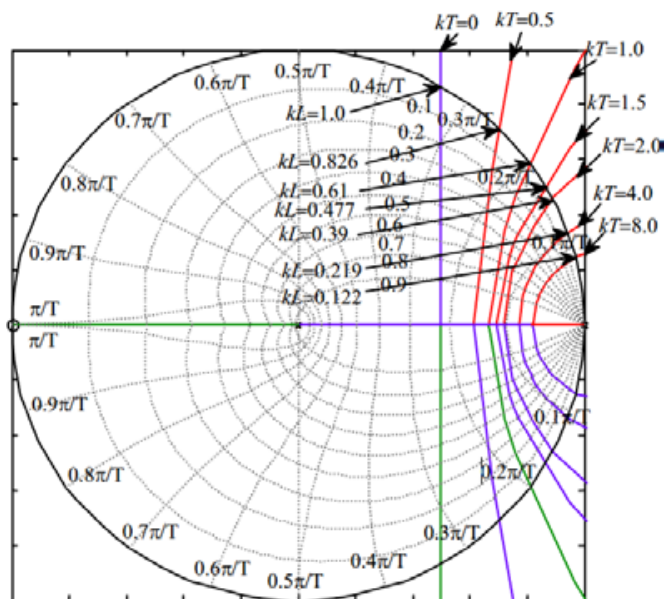

Picture 3 Orbit Diagram of Different Roots in the Change Process of $k_{\mathrm{T}}$ Value from 0 to 8

Picture 3 gives the orbit diagram of different roots in the change process of kT value from 0 to 8 . Within the stable range, comparing with one-step-delay control, following the increase of delay coefficient $\mathrm{kT}$, the upper limit value of kT gradually decreases, that is the required inductance value is smaller and smaller. The decrease of kT will lower the control precision of system and influence the stability of grid-connected inverting system.

\section{Experimental Results and Analysis}

In order to verify the superiority of the proposed grid-connected inverter robust prediction dead-beat control method in lifting system stability, the feasibility analysis of robust dead-beat method is performed in 35kW PV grid-connected inverting experimental platform shown in Picture 4.. It is shown in Picture 6 that power feedforward and robust current dead-beat control are added which are used for strengthening system response performance and lowering sensitivity of parameters respectively.

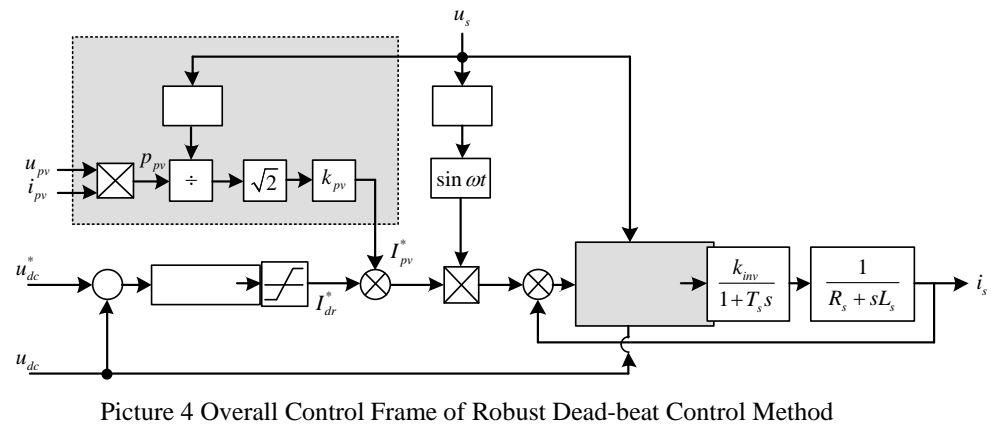



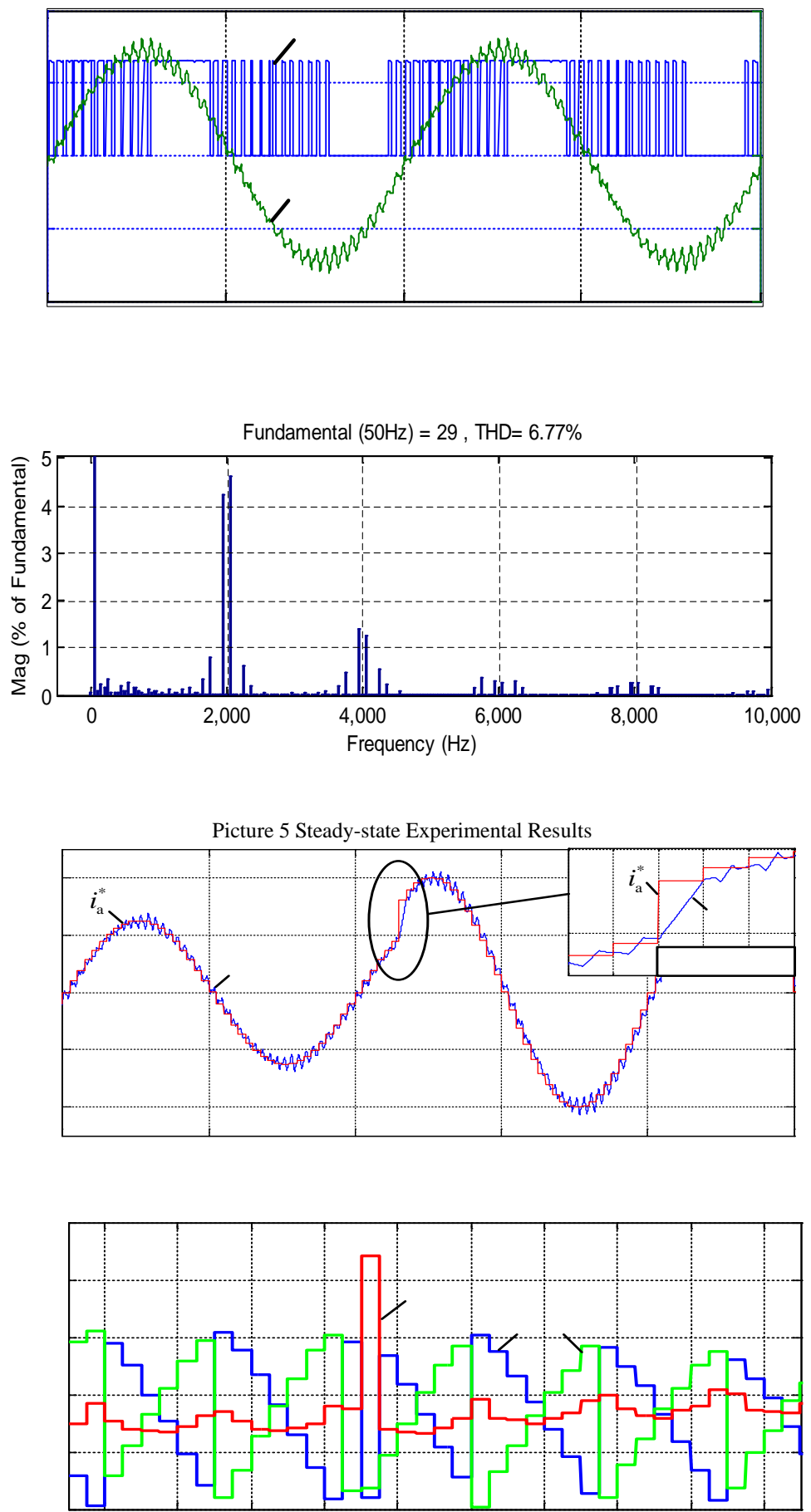

Picture 6 Dynamic Experimental Results

\section{Conclusion}

This article studies a power feedforward robust prediction dead-beat grid-connected control method. The following conclusions can be got through relative theories analysis and model machine experimental verification: 1) The conclusion that current control precision and system stability of traditional dead-beat control is easy to be influenced by mismatching of delay and filter inductance value is gained by performing stability analysis on Z-domain dead-beat control model with delay link. 2) Using robust prediction current dead-beat control can effectively overcome instability of system caused by digital system delay, parameter matching and so on which can further lift the actual application significance of dead-beat control. 


\section{Reference}

[1]Zhong, Chen, Stefan Müller Arisona, Xianfeng Huang, Michael Batty, and Gerhard Schmitt. "Detecting the dynamics of urban structure through spatial network analysis." International Journal of Geographical Information Science 28, no. 11 (2014): 2178-2199.

[2]Li, Wubin, Johan Tordsson, and Erik Elmroth. "An aspect-oriented approach to consistency-preserving caching and compression of web service response messages." In Web Services (ICWS), 2010 IEEE International Conference on, pp. 526-533. IEEE, 2010.

[3]Y. Geng, J. He, K. Pahlavan, Modeling the Effect of Human Body on TOA Based Indoor Human Tracking[J], International Journal of Wireless Information Networks 20(4), 306-317

[4]Lv, Zhihan, and Tianyun Su. "3D seabed modeling and visualization on ubiquitous context." In SIGGRAPH Asia 2014 Posters, p. 33. ACM, 2014. 